\title{
FORCE FREE MÖBIUS MOTIONS OF THE CIRCLE
}

\author{
DANIELA EMMANUELE AND MARCOS SALVAI
}

Communicated by Izu Vaisman

Abstract. Let $\mathcal{M}$ be the Lie group of Möbius transformations of the circle. Suppose that the circle has initially a homogeneous distribution of mass and that the particles are allowed to move only in such a way that two configurations differ in an element of $\mathcal{M}$. We describe all force free Möbius motions, that is, those curves in $\mathcal{M}$ which are critical points of the kinetic energy. The main tool is a Riemannian metric on $\mathcal{M}$ which turns out to be not complete (in particular not invariant, as happens with non-rigid motions) given by the kinetic energy.

\section{Introduction}

In the spirit of the classical description of the force free motions of a rigid body in Euclidean space using an invariant metric on SO (3) [1, Appendix 2], the second author defined in [4] an appropriate metric on the Lorenz group $\mathrm{SO}_{o}(n+1,1)$ to study force free conformal motions of the sphere $\mathbb{S}^{n}$, obtaining a few explicit ones (only through the identity and those which can be described using the Lie structure of the configuration space). In this note, in the particular case $n=1$, that is, Möbius motions of the circle, we obtain all force free motions.

This is an example of a situation in which using concepts of Physics one can state and solve a problem in Differential Geometry (see for instance $[2,3,6]$ ).

Notice that the canonical action of $\operatorname{PSL}(2, \mathbb{R})$ on $\mathbb{R} \mathbb{P}^{1} \cong \mathbb{S}^{1}$ is equivalent to the action of the group of Möbius transformations on the circle. Then, the results presented here, up to a double covering, also extend the case $n=1$ of [5], where force free projective motions of the sphere $\mathbb{S}^{n}$ were studied.

This note, as well as [4,5], is weakly related with mass transportation [7]. In our situation, the set of admitted mass distributions is finite dimensional, and also the allowed transport maps are very particular. 


\subsection{Möbius Motions of the Circle}

Let $\mathbb{S}^{1}$ be the unit circle centered at zero in $\mathbb{C}$ with the usual metric and let $\mathcal{M}$ be the Lie group of Möbius transformations of the circle, that is, the group of Möbius transformations of the extended plane preserving the circle. It consists of maps of the form $c T_{\alpha}$, where $c \in \mathbb{S}^{1}$ and

$$
T_{\alpha}(z)=\frac{z+\alpha}{1+\bar{\alpha} z}
$$

for $\alpha \in \mathbb{C},|\alpha|<1$ and all $z \in \mathbb{S}^{1}$. Although we are interested in the action of $\mathcal{M}$ on the circle, we recall that if the unit disc $\Delta=\{z \in \mathbb{C} ;|z|<1\}$ carries the canonical Poincaré metric of constant negative curvature -1 and $\alpha \neq 0$, then $T_{\alpha}$ is the transvection translating the geodesic with end points $\pm \alpha /|\alpha|$, which sends 0 to $\alpha$.

A Möbius motion of the circle is by definition a smooth curve in $\mathcal{M}$, thought of as a curve of diffeomorphisms of the circle. (Throughout the paper, smooth means of class $C^{\infty}$.)

In the next two subsections we recall, specialized for the circle, some definitions and statements given in [4] for conformal motions on the $n$-dimensional sphere.

\subsection{The Energy of Möbius Motions of Circle}

Suppose that the circle has initially a homogeneous distribution of mass of constant density one and that the particles are allowed to move only in such a way that two configurations differ in an element of $\mathcal{M}$. The configuration space may be naturally identified with $\mathcal{M}$.

Let $\gamma:\left[t_{0}, t_{1}\right] \rightarrow \mathcal{M}$ be a Möbius motion of $\mathbb{S}^{1}$. The total kinetic energy $E_{\gamma}(t)$ of the motion $\gamma$ at the instant $t$ is given by

$$
E_{\gamma}(t)=\frac{1}{2} \int_{\mathbb{S}^{1}}\left|v_{t}(q)\right|^{2} \rho_{t}(q) \mathrm{d} m(q)
$$

where integration is taken with respect to the canonical volume form of $\mathbb{S}^{1}$ and, if $q=\gamma(t)(p)$ for $p \in \mathbb{S}^{1}$, then

$$
v_{t}(q)=\left.\frac{\mathrm{d}}{\mathrm{d} s}\right|_{t} \gamma(s)(p) \in T_{q} \mathbb{S}^{1}, \quad \rho_{t}(q)=1 / \operatorname{det}\left(\mathrm{d} \gamma(t)_{p}\right)
$$


are the velocity of the particle $q$ and the density at $q$ at the instant $t$, respectively. Applying to (2) the formula for change of variables, one obtains

$$
E_{\gamma}(t)=\left.\frac{1}{2} \int_{\mathbb{S}^{1}}\left|\frac{\mathrm{d}}{\mathrm{d} s}\right|_{t} \gamma(s)(p)\right|^{2} \mathrm{~d} m(p) .
$$

The kinetic energy of $\gamma$ is defined by

$$
E(\gamma)=\int_{t_{0}}^{t_{1}} E_{\gamma}(t) \mathrm{d} t
$$

The following definition is based on the principle of least action.

Definition 1. A smooth curve $\gamma$ in $\mathcal{M}$, thought of as a Möbius motion of $\mathbb{S}^{1}$, is said to be force free if it is a critical point of the kinetic energy functional, that is,

$$
\left.\frac{\mathrm{d}}{\mathrm{d} s}\right|_{0} E\left(\gamma_{s}\right)=0
$$

for any proper smooth variation $\gamma_{s}$ of $\gamma\left(\right.$ here $\gamma_{s}(t)=\Gamma(s, t)$, where $\Gamma:(-\varepsilon, \varepsilon) \times$ $\left[t_{0}, t_{1}\right] \rightarrow \mathcal{M}$ is a smooth map, with $\varepsilon>0, \Gamma(0, t)=\gamma(t)$ and $\Gamma\left(s, t_{i}\right)=\gamma\left(t_{i}\right)$ for all $s \in(-\varepsilon, \varepsilon), i=0,1)$.

\subsection{A Riemannian Metric on the Configuration Space}

Given $g \in \mathcal{M}$ and $X \in T_{g} \mathcal{M}$, let us define the map $\tilde{X}: \mathbb{S}^{1} \rightarrow T \mathbb{S}^{1}$ by

$$
\widetilde{X}(q)=\left.\frac{\mathrm{d}}{\mathrm{d} t}\right|_{0} \gamma(t)(q) \in T_{g(q)} \mathbb{S}^{1}
$$

where $\gamma$ is any smooth curve in $\mathcal{M}$ with $\gamma(0)=g$ and $\dot{\gamma}(0)=X$. The map $\tilde{X}$ is well-defined and smooth and it is a vector field on $\mathbb{S}^{1}$ if and only if $X \in T_{e} \mathcal{M}$. Moreover,

$$
X \mapsto\|X\|^{2}=\frac{1}{2 \pi} \int_{\mathbb{S}^{1}}|\widetilde{X}(q)|^{2} \mathrm{~d} m(q)
$$

is a quadratic form on $T_{g} \mathcal{M}$ and gives a Riemannian metric on $\mathcal{M}$.

Remarks 2. a) The fundamental property of the metric (5) on $\mathcal{M}$ is that a curve $\gamma$ in $\mathcal{M}$ is a geodesic if and only if (thought of as a Möbius motion) it is force free, since by (5) and (3), $E_{\gamma}(t)=\pi\|\dot{\gamma}(t)\|^{2}$.

b) The metric on $\mathcal{M}$ is neither left nor right invariant, since we saw in [4] that it is not even complete. 


\section{Force Free Möbius Motions of the Circle}

The next theorem describes completely the geometry of $\mathcal{M}$ endowed with the metric (5) given by the kinetic energy. Recall from (1) that $T_{a}$ denotes the transvection associated with $\alpha$ and that $\Delta$ is the unit disc centered at zero in $\mathbb{C}$.

Theorem 3. Let $\mathrm{d} s^{2}$ be the metric on the disc $\Delta$ given in polar coordinates $(r, \theta)$ by

$$
\mathrm{d} s^{2}=\frac{2\left(\mathrm{~d} r^{2}+r^{2} \mathrm{~d} \theta^{2}\right)}{1-r^{2}}
$$

and consider on $\mathbb{S}^{1} \times \Delta$ the Riemannian product metric, where $\mathbb{S}^{1}$ has length $2 \pi$. Then the map

$$
F: \mathbb{S}^{1} \times \Delta \rightarrow \mathcal{M}, \quad F(u, \alpha)=u T_{\alpha}
$$

is an isometry.

Remarks 4. a) Note that the metric (6) on $\Delta$ is not the canonical metric of constant negative curvature on $\Delta$. Indeed, the curvature function can be easily computed to be $K(r, \theta)=-1 /\left(1-r^{2}\right)$, in particular, it tends to $-\infty$ as $r \rightarrow 1^{-}$. Also, the metric on $\Delta$ is not complete, since the inextendible ray $(0,1)$ э $r \mapsto T_{r}$ has length $\pi / \sqrt{2}$, since $\left\|\frac{\partial}{\partial r}\right\|^{2}=\frac{2}{1-r^{2}}$.

b) In the higher dimensional situation [4] it is proven that the group $\mathrm{SO}(n)$ (with the metric induced from the one given by the kinetic energy) is totally geodesic in the group of directly conformal transformations of $\mathbb{S}^{n}$, but the author did not know whether this subgroup is a Riemannian factor, as it turned to be for $n=1$. In the projective case [5], $\mathrm{SO}(n)$ is not even totally geodesic.

Proof of Theorem 3. Let $\mathbb{S}^{1} \subset \mathcal{M}$ be the subgroup of isometries of the circle. The torus $\mathbb{S}^{1} \times \mathbb{S}^{1}$ acts on $\mathcal{M}$ on the left by $(u, v) \cdot g=u g \bar{v}$, where $(u g \bar{v})(z)=u g(z \bar{v})$ for any $z \in \mathbb{S}^{1}$. We know from the higher dimensional cases in [4] that this action is by isometries of $\mathcal{M}$, provided that this group is endowed with the metric (5).

We fix $0<r<1$. By the torus symmetry just described, it suffices to verify that $\mathrm{d} F_{(1, r)}: T_{(1, r)}\left(\mathbb{S}^{1} \times \Delta\right) \rightarrow T_{F(1, r)} \mathcal{M}$ is a linear isometry. We put coordinates $t \mapsto \mathrm{e}^{\mathrm{i} t}$ on $\mathbb{S}^{1}$ and $(\rho, \theta) \mapsto \rho \mathrm{e}^{\mathrm{i} \theta}$ on $\Delta$. We denote $\partial_{x}=\frac{\mathrm{d}}{\mathrm{d} x}$. Let $X, Y, Z$ be the images under $\mathrm{d} F_{(1, r)}$ of $\partial_{t}, \partial_{\rho}, \partial_{\theta}$, respectively. It suffices to show that $\{X, Y, Z\}$ is an orthogonal basis of $T_{F(1, r)} \mathcal{M}$ with

$$
\|X\|^{2}=1, \quad\|Y\|^{2}=\frac{2}{1-r^{2}}, \quad\|Z\|^{2}=\frac{2 r^{2}}{1-r^{2}} .
$$


First, we compute $\widetilde{X}, \widetilde{Y}$ and $\widetilde{Z}$ by their definition (4). In each case, we take the curve $\gamma$ as the image under $F$ of the coordinate curves in $\mathbb{S}^{1} \times \Delta$ through the point $(1, r)$. We have

$$
\begin{aligned}
\widetilde{X}(z) & =\left.\frac{\mathrm{d}}{\mathrm{d} t}\right|_{0} F\left(\mathrm{e}^{\mathrm{i} t}, r\right)(z)=\left.\frac{\mathrm{d}}{\mathrm{d} t}\right|_{0} \mathrm{e}^{\mathrm{i} t} T_{r}(z)=\left.\mathrm{i} \mathrm{i}^{\mathrm{i} t} T_{r}(z)\right|_{t=0}=\mathrm{i} T_{r}(z)=\mathrm{i} \frac{z+r}{1+r z} \\
\widetilde{Y}(z) & =\left.\frac{\mathrm{d}}{\mathrm{d} \rho}\right|_{r} F(1, \rho)(z)=\left.\frac{\mathrm{d}}{\mathrm{d} \rho}\right|_{r} T_{\rho}(z)=\left.\frac{\mathrm{d}}{\mathrm{d} \rho}\right|_{r} \frac{z+\rho}{1+\rho z}=\frac{1-z^{2}}{(1+r z)^{2}} \\
\widetilde{Z}(z) & =\left.\frac{\mathrm{d}}{\mathrm{d} \theta}\right|_{0} F\left(1, r \mathrm{e}^{\mathrm{i} \theta}\right)(z)=\left.\frac{\mathrm{d}}{\mathrm{d} \theta}\right|_{0} T_{r \mathrm{e}} \mathrm{i} \theta(z)=\left.\frac{\mathrm{d}}{\mathrm{d} \theta}\right|_{0} \frac{z+r \mathrm{e}^{\mathrm{i} \theta}}{1+r \mathrm{e}^{-\mathrm{i} \theta} z} \\
& =\frac{\mathrm{i} r\left(1+2 r z+z^{2}\right)}{(1+r z)^{2}} .
\end{aligned}
$$

Next we compute

$$
2 \pi\|X\|^{2}=\int_{\mathbb{S}^{1}}|\tilde{X}(z)|^{2} \mathrm{~d} m(z)=\int_{\mathbb{S}^{1}}\left|\mathrm{i} T_{r}(z)\right|^{2} \mathrm{~d} m(z)=\int_{\mathbb{S}^{1}} 1 \mathrm{~d} m(z)=2 \pi .
$$

We have also

$$
2 \pi\|Y\|^{2}=\int_{\mathbb{S}^{1}}|\widetilde{Y}(z)|^{2} \mathrm{~d} m(z)=\int_{\mathbb{S}^{1}}\left|\frac{1-z^{2}}{(1+r z)^{2}}\right|^{2} \mathrm{~d} m(z) .
$$

Setting $z=\mathrm{e}^{\mathrm{i} s}$, we have

$$
2 \pi\|Y\|^{2}=\int_{0}^{2 \pi} \frac{1}{\mathrm{ie}^{\mathrm{i} s}}\left|\frac{1-\mathrm{e}^{\mathrm{i} 2 s}}{\left(1+r \mathrm{e}^{\mathrm{i} s}\right)^{2}}\right|^{2} \mathrm{ie}^{\mathrm{i} s} \mathrm{~d} s=\int_{\mathbb{S}^{1}} \frac{1}{\mathrm{i} z}\left|\frac{1-z^{2}}{(1+r z)^{2}}\right|^{2} \mathrm{~d} z .
$$

Now, the integrand is a complex analytic function inside the circle (observe that $\bar{z}=1 / z$ for $|z|=1$ ), except for a simple pole at $z=0$ and a pole of order two at $z=-r$, with residues $\frac{\mathrm{i}}{r^{2}}$ and $\frac{\mathrm{i}\left(r^{2}+1\right)}{-r^{2}\left(1-r^{2}\right)}$, respectively. One obtains that $\|Y\|^{2}=2 /\left(1-r^{2}\right)$. In the same way one gets $\|Z\|^{2}=2 r^{2} /\left(1-r^{2}\right)$.

We claim that the vectors $X, Y, Z$ are pairwise orthogonal. Let $h(U, V)=U \bar{V}$ denote the Hermitian inner product on $\mathbb{C}$. We compute

$$
\int_{\mathbb{S}^{1}} h(\widetilde{X}(z), \widetilde{Y}(z)) \mathrm{d} m(z)=\int_{\mathbb{S}^{1}} f(z) \mathrm{d} z
$$

where $f(z)=\frac{z^{2}-1}{z(1+r z)(z+r)}$ is an complex analytic function inside the circle, except for simple poles at $z=0$ and $z=-r$, with residues $1 / r$ and $-1 / r$, respectively. Then,

$$
\langle X, Y\rangle=\Re \int_{\mathbb{S}^{1}} h(\tilde{X}(z), \tilde{Y}(z)) \mathrm{d} m(z)=0 .
$$


Analogously, we find that $\langle Y, Z\rangle=\langle X, Z\rangle=0$.

Corollary 5. The force free Möbius motions of the circle, or equivalently, the geodesics of $\mathcal{M}$, are, via $F$, of the form $\gamma=\left(\gamma_{1}, \gamma_{2}\right)$, where $\gamma_{1}$ parametrizes the circle with constant speed and $\gamma_{2}$ is a geodesic in the disc $\Delta$ whose trajectory coincides with the images of either $c_{1}(\rho)=\left(\rho, \theta_{0}\right)$ or $c_{2}(\theta)=(\rho(\theta), \theta)$, where $\rho$ satisfies the differential equation

$$
\left(\rho^{\prime}\right)^{2}=\frac{\mu+\rho^{2}}{\left(1-\rho^{2}\right) \rho^{2}}
$$

for some constant $\mu>-1$.

Proof. Clearly, a geodesic of a Riemannian product projects to a geodesic in each factor. Besides, as the coefficients of the first fundamental form of $\Delta$ depend only on $\rho$, the corresponding metric is Clairaut. Then, the trajectories of the geodesics of $\Delta$ are, in polar form

$$
c_{1}(\rho)=\left(\rho, \theta_{0}\right) \quad \text { or } \quad c_{2}(\theta)=(\rho(\theta), \theta)
$$

for some constant $\theta_{0}$, where $\rho(\theta)$ satisfies Clairaut's differential equation, for some constant $\lambda$

$$
\lambda E^{2}(\rho)=E(\rho)+\left(\rho^{\prime}\right)^{2} G(\rho) .
$$

Since in our case $E(\rho)=\left\|\frac{\partial}{\partial r}\right\|^{2}=\frac{2}{1-\rho^{2}}$ and $G(\rho)=\left\|\frac{\partial}{\partial \theta}\right\|^{2}=\frac{2 \rho^{2}}{1-\rho^{2}}$, the differential equation is equivalent to (7) for some constant $\mu>-1$.

\section{Acknowledgements}

This work is partially supported by FONCYT, CIEM (CONICET), SECYT (UNC) and SECYT (UNR).

\section{References}

[1] Arnold V., Mathematical Methods of Classical Mechanics, Graduate Texts in Math. vol. 60, Springer, Berlin 1989.

[2] Coulton P., Foote R. and Galperin G., The Dynamics of Pendulums on Surfaces of Constant Curvature, Math. Phys. Anal. Geom. 12 (2009) 97-107.

[3] Salvai M., On the Dynamics of a Rigid Body in the Hyperbolic Space, J. Geom. Phys. 36 (2000) 126-139. 
[4] Salvai M., Force Free Conformal Motions of the Sphere, Diff. Geom. Appl. 16 (2002) 285-292.

[5] Lazarte M., Salvai M. and Will A., Force Free Projective Motions of the Sphere, J. Geom. Phys. 57 (2007) 2431-2436.

[6] Nagy P., Dynamical Invariants of Rigid Motions on the Hyperbolic Plane, Geom. Dedicata 37 (1991) 125-139.

[7] Villani C., Topics in Optimal Transportation, Graduate Studies in Mathematics vol. 58, American Mathematical Society, Providence 2003.

Daniela Emmanuele

Departamento de Matemática

Escuela de Ciencias Exactas y Naturales

FaCEIA

Universidad Nacional de Rosario

2000 Rosario, ARGENTINA

E-mail address: emmandfceia.unr.edu.ar

Marcos Salvai

FaMAF - CIEM, Ciudad Universitaria

5000 Córdoba, ARGENTINA

E-mail address: salvaiefamaf . unc.edu.ar 